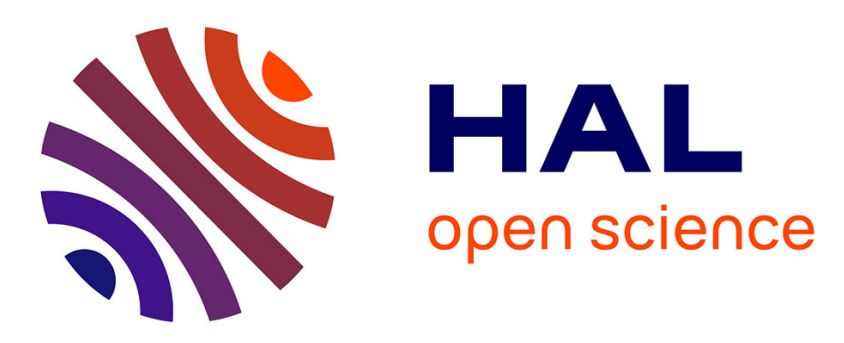

\title{
Simulation Data Management and Reuse: Toward a Verification and Validation Approach
}

\author{
Anaïs Ottino, Thomas Vosgien, Julien Le Duigou, Nicolas Figay, Pascal \\ Lardeur, Benoît Eynard
}

\section{- To cite this version:}

Anaïs Ottino, Thomas Vosgien, Julien Le Duigou, Nicolas Figay, Pascal Lardeur, et al.. Simulation Data Management and Reuse: Toward a Verification and Validation Approach. 12th IFIP International Conference on Product Lifecycle Management (PLM), Oct 2015, Doha, Qatar. pp.476-484, 10.1007/978-3-319-33111-9_43 . hal-01377473

\section{HAL Id: hal-01377473 \\ https://hal.inria.fr/hal-01377473}

Submitted on 7 Oct 2016

HAL is a multi-disciplinary open access archive for the deposit and dissemination of scientific research documents, whether they are published or not. The documents may come from teaching and research institutions in France or abroad, or from public or private research centers.
L'archive ouverte pluridisciplinaire HAL, est destinée au dépôt et à la diffusion de documents scientifiques de niveau recherche, publiés ou non, émanant des établissements d'enseignement et de recherche français ou étrangers, des laboratoires publics ou privés.

\section{(c)(1)}

Distributed under a Creative Commons Attribution| 4.0 International License 


\title{
Simulation data management and reuse: toward a verification and validation approach
}

\author{
Anaïs Ottino ${ }^{1,2}$, Thomas Vosgien ${ }^{2}$, Julien Le Duigou ${ }^{1}$, Nicolas Figay ${ }^{2}$, Pascal \\ Lardeur $^{1}$, Benoît Eynard ${ }^{1}$ \\ ${ }^{1}$ Laboratoire Roberval, Université de Technologie de Compiègne, Sorbonne Universités, \\ Compiègne, France \\ anais.ottino@utc.fr; julien.le-duigou@utc.fr; pascal.lardeur@utc.fr; benoit.eynard@utc.fr \\ ${ }^{2}$ Institut de Recherche Technologique SystemX, Université Paris-Saclay, Palaiseau, France \\ anais.ottino@irt-systemx.fr; thomas.vosgien@irt-systemx.fr; nicolas.figay@irt-systemx.fr
}

\begin{abstract}
Nowadays, in various sectors of industry, numerical simulation process becomes more and more time consuming. In this process, the lead time of the pre-processing stage is predominant. Therefore, in order to optimize this process and hence, the design process, the created computational models need to be reused. According to Product Lifecycle Management (PLM) approach in an extended enterprise context, the computational models come from various partners, departments and heterogeneous tools. In order to reuse these computational models, it is necessary to capitalize the simulation data in accordance with a common standardized and structured format. Based on a Simulation Lifecycle Management (SLM) approach and a Verification \& Validation methodology, this paper proposes a framework and a process to enable the reuse of computational models.
\end{abstract}

Keywords: Numerical simulation, STEP AP209, Simulation Lifecycle Management, Verification \& Validation

\section{Introduction}

In the industry, projects progress through partnerships. These partnerships involve the generation of a large amount of data form various sources throughout the product lifecycle [1]. This collaborative context implies the necessity to manage all the data concerning the product used by the different partners through various activities. It describes the Product Lifecycle Management (PLM) approach [2]. The "Standard \& Interoperability PLM" ${ }^{1}$ project, which includes this research work, aims at developing an experimental platform as a Service. The objective of the platform is to validate the usage and the implementation of PLM standards through industrial scenarios [3].

Today, the highly competitive business environment pushes companies to deliver more innovative products, reduce costs, improve quality, and shorten time to market.

\footnotetext{
${ }^{1}$ http://www.irt-systemx.fr/project/sip/
} 
The increasing complexity of products adds difficulties. To support design decisions and to better understand product behavior, simulation and analysis is becoming increasingly important to manufacturing enterprises [4]. Simulation analyses are often a combination of physical disciplines and based on heterogeneous technologies. To compete on the global market, companies focus on new approaches like the Simulation Lifecycle Management (SLM) and design for product variation in order to rapidly achieve product quality and process robustness [5].

This context accentuates the need to establish an effective SLM approach though the product development process. The National Agency for the Finite Element Methods and Standards (NAFEMS) defines the SLM approach as "management of the intellectual property associated with simulation tools, data, and processes as related to product or process development”. A Simulation Data Management (SDM) system supports the SLM approach. It must encompass four essential functional areas to be effective: collaboration, data structuration, decision support, and integration and process automation [6].

The simulation and modeling process is classically composed of three steps: the pre-processing step, the computation step and the post-processing step. Most of the time consumed by this process is gathered on the pre-processing step, in other words, the creation of the computational model. In a collaborative environment, a number of gaps have been identified in the simulation process by [7]. There is loss of information during the acquisition of input data and during the idealization of models leading to rework activities. In addition, time loss results from the computational models already created which are not reused in the simulation processes. In some industry area, the simulation teams are isolated by business field and there is limited communication. Yet, the global optimization of a product must be result from a multidisciplinary and multi-physics compromise. It is necessary to exchange information from different disciplines during the product development process for managing the various phenomena influence.

Our study is placed in an industrial context where a new product development takes place in an extended enterprise. This organizing principle allows the initiated enterprise to cooperate through alliances and partnerships. The objectives of this collaboration is to carry out activities without all the necessary competencies and/or without the necessary internal resources. In this context, there is three possible types of reuse of computational model: in the same simulation department, in another simulation department and in a partner company. These three types of reuse imply an intra-physical and/or inter-physical simulation data exchange.

The objective of this paper is to propose a business process based on several existing approaches for setting up the reuse of computational models, in a collaborative and multidisciplinary context. The study presents how the combination of the SLM approach and the V\&V methodology enable the reuse of computational models. 


\section{SLM approach, $V \& V$ and standards of data exchanges}

Like presented in the introduction, the SLM approach implies the effectivity of four areas: collaboration, data model, decision support, and integration and process automation. This work aims to enhance the use of SDM systems for reuse of computational models. In this objective, the $\mathrm{V} \& \mathrm{~V}$ methodology allows to provide a set of information about the models. To address the issue of product development in a collaborative and multi-partner environment, it is necessary to exchange the simulation data and the resulting computational model in accordance with international standards. The use of international standards is important to establish an unambiguous "language" between partners in order to ensure product definition consistency throughout its lifecycle [8]. This section presents in a first part the research works connecting the SLM approach and the V\&V methodology. The second part deals with the description of the $\mathrm{V} \& \mathrm{~V}$ methodology and the third part introduces the existing standards for the simulation data exchange.

\subsection{SLM approach and V\&V methodology}

Lots of research works had demonstrated the interest for the enterprises to adopt an harmonized SLM approach and collaboration process for product development [9-13]. The SLM approach is identified by [6] as a component of the PLM approach. Given the particularity of data, process and lifecycle, the numerical simulation needs its own approach.

The state of the art shows a set of works concerning the V\&V methodology in the product development process, the data management, and the collaboration process with the objective to enhance the lead time and the product reliability. The simulation lifecycle is defined as an iterative and reversible phases associated with a V\&V activity [11]. According to the author, $V \& V$ is not a step or a phase in the lifecycle but a continuous activity through the entire simulation lifecycle. An approach based on V\&V methodology applied on digital mock-up in the system engineering field for the "International Thermonuclear Experimental Reactor" (ITER) project had been proposed by [12]. The ITER project aims to demonstrate the reliability and the workability of a fusion reactor. The authors formalize the design process in a PLM/SLM context according to the $\mathrm{V} \& \mathrm{~V}$ process in order to enhance costs and reliability of the ITER remote handling systems. Their study uses the V\&V methodology for complex system engineering and highlights the importance of PLM in this approach. Several methods and technics linked with the design V\&V methodology in the product lifecycle have been gathered by [13]. Their analysis was about standardized definitions of $\mathrm{V} \& \mathrm{~V}$ concepts in design context. They gave activity and process classification, from preliminary design to physical $\mathrm{V} \& \mathrm{~V}$ step, in the production phase. 


\subsection{The V\&V methodology}

In a reuse context, the model is created by another person than the user. Consequently, it is important for the user to have information allowing the determination of the reuse possibility or not, according to his simulation scenario. The $\mathrm{V} \& \mathrm{~V}$ methodology associated with the modeling and simulation process allows to get a set of model information called data $\mathrm{V} \& \mathrm{~V}$. In computational physics, the partial differential equations are used to formulate problems and describe a wide variety of phenomena. The $\mathrm{V} \& \mathrm{~V}$ methodology is used preferably on computational models being based upon methods allowing to solve these equations (such as finite element method, finite difference method, and finite volume method). To introduce the V\&V methodology, it is necessary to give a definition of computational model. The computational model is a mathematical and numerical description of a specific simulation scenario including geometric data, material characteristics, and information about initial and boundary conditions [14].

The Society for Computer Simulation (SCS) Technical Committee on Model Credibility proposed in the late 1970 s, a simplified vision of V\&V process in order to highlight the interactions between evaluation phases [15]. The figure 1 presents an adaptation of this process made by [14] and [16] for $\mathrm{V} \& \mathrm{~V}$ of computational models.

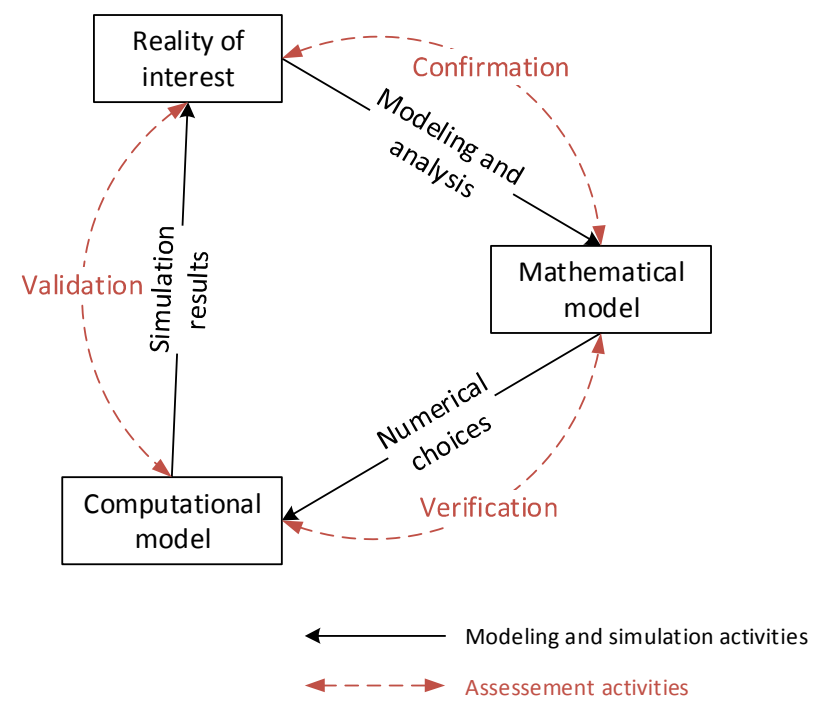

Fig. 1. Simplified vision of $V \& V$ process phases of computational models adapted from Schlesinger [15] by Thacker et al. [14] and Oberkampf et al. [16]

The reality of interest is the physical system on which a simulation demand is made about a specific problem to solve. It is analyzed to create the mathematical model. The mathematical model encompasses physical assumptions, mathematical 
equations, and physical modeling data (boundary conditions, loading, law of material behavior). The computational model is the implementation of the mathematical model associated with a numerical approximation and a convergence criterion [17].

The assessment phases of the process consist in "solving the equations right" (verification phase) and "solving the right equations" (validation phase) [18]. The verification phase aims to compare a reference solution of the mathematical model with the numerical solution obtained with the computation model. The validation phase aims to compare the solution of computational model, identified during the verification phase, with the reality of interest. The validation process is conclusive when the difference between the experimental and the numerical results are judged satisfactory [17].

\subsection{Standards for data exchange in numerical simulation}

The standards are used to preserve collected data during the product development in a collaborative environment. The interoperability assessment of business applications, associated with a verification capacity according to consistent standard, become a challenge for manufacturing industries. In the numerical simulation, several standards exist for exchange data and information, each one specialized for specific business needs. The CFD General Notation System (CGNS) standard covers the computational fluid dynamics data [19]. The objective of CGNS is to facilitate the data exchange between applications and to make durable aerodynamics data archiving. The STEP-Thermal Analysis for Space (STEP-TAS) standard [20] allows exchange, processing, and archiving in the long run, of models and thermal analysis results for space. The ISO10303 STEP-AP209 second edition standard covers multidisciplinary design and analysis [21]. It deals with geometric aspects, analysis with finite element method and the computational fluid dynamics. The ISO10303 STEP-AP209 is an application protocol of ISO10303 STEP family[22]. The ISO10303 STEP standard is a set of application protocols covering the product representation and data exchange needed for its description throughout the life cycle.

The figure 2 illustrates the functional coverage of the ISO10303 STEP-AP209 standard. The left side identifies the common application modules with the ISO10303 STEP-AP242 application protocol. The application protocol 242 deals with the management of model-based 3D engineering [23]. The right side identifies the specific 209 application modules. The use of ISO10303 STEP-AP209 standard, like common information model for exchange and manage simulation data, is entered into a SLM approach. However it is currently not enough to represent and exchange V\&V data. 


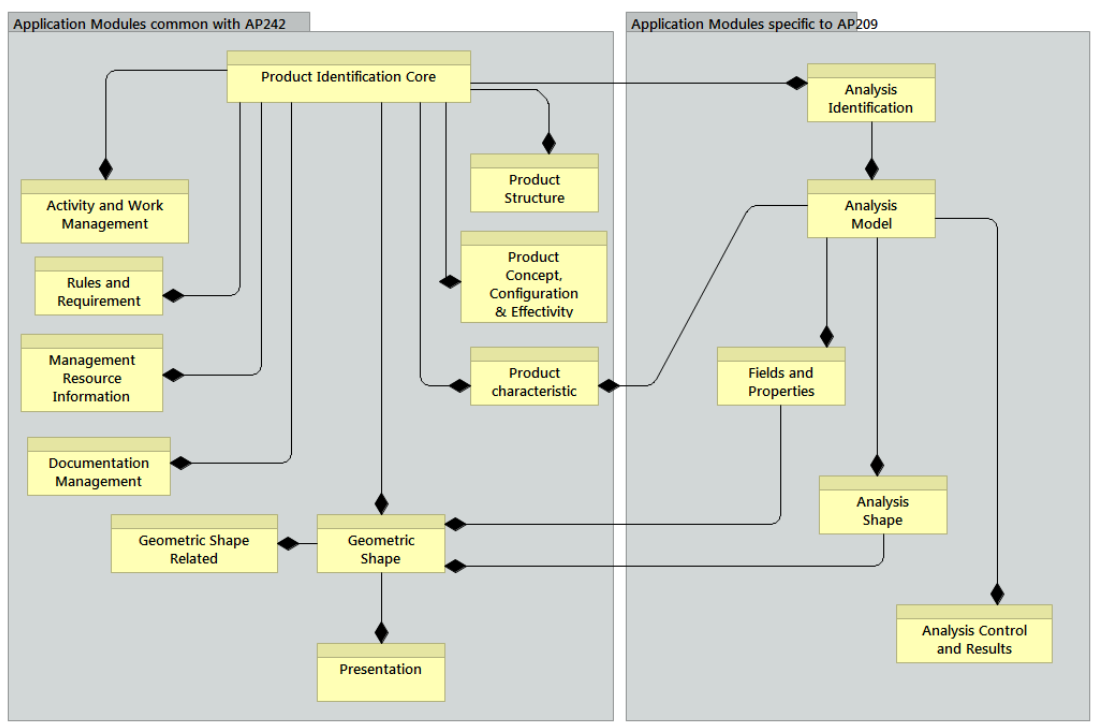

Fig. 2. Modeling of the ISO10303 STEP-AP209 functional coverage adapted from [24]

Recommended practice guides have been produced in order to implement V\&V process in companies: the ARP 755A [25] in the aeronautics field and the ASME V V 10 [26] in the solid mechanics field. Standards on V\&V have been also developed in various fields. The 1012 standard developed by IEEE SA covers V\&V process in the software engineering field [27]. It aims to develop, maintain, and reuse systems, software, and hardware. The V V 20 standard developed by ASME covers the fluid mechanics field [28]. It quantifies the accuracy degree of a model by comparing the numerical and the experimental results for one variable at one specified validation point. The ISO10303 STEP-AP233 standard for system engineering includes also a set of application modules for the management of requirements and its verification and validation [29].

According to existing state of the art and standards, the figure 3 shows simulation data which must be integrated throughout the simulation lifecycle. 


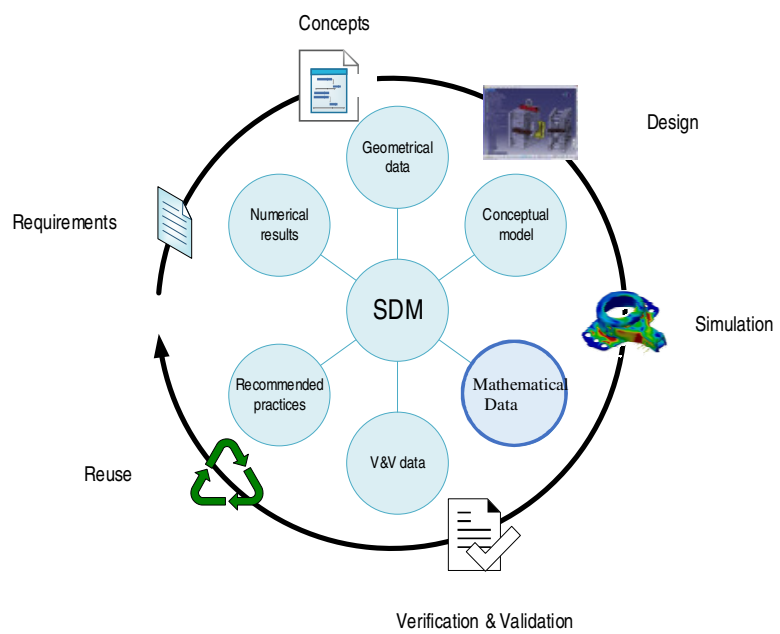

Fig. 3. Simulation lifecycle and data management linked to the model.

\section{Reuse process of computational models}

The reuse of computational model is the selection, the adaptation, and the use of this model for a new objective. During the modeling and simulation process, a set of activities are sequentially made, generating transitional models from the extraction of the digital mock-up to the finite element model.

The figure 4 proposes a simulation process with the possibility to reuse the models. The boxes represent the business functions and in some cases the associate business objects. The arrows represent the data flows. The red boxes are business function linked to an assessment activity. The language used in figure 4 is an enterprise architecture modeling language called ArchiMate [30].

Figure 4 presents the process allowing early reuse of models. The analysis of simulation requirements allows to define the simulation objectives and the validation requirements. The specification step is used for define physical hypothesis, mathematical equations, and physical data of modeling. This definition and specification phase of the simulation problem enables the establishment of a scope statement. The request activity of existing models permits to test the SDM on the reuse possibility of already created models. A distance criterion is used to select the closest model at the more advanced step (CAD, idealized model, mesh model...). This criterion encompasses verification information and is used in a request to the SDM system to get the best model to reuse.

In accordance with the answer to the SDM request, the process is divided in two branches. The first branch corresponds to the classic simulation process. As no relevant model has been found, a new model is created. The second branch corresponds to the case where a close existing model is found in the SDM. In this case, it is necessary to export the model from the SDM, and, if needed, transform data 
to recipient format, and adapt the model for solving the specific problem. Each activity, on the two process branches, generates transitional models which are subjected to a verification step. The verification activity depends on the business activity. The verification data enrich the SDM and make the reuse process more efficient. All the needed information can be store in the SDM as the data model (STEP AP209ed2) is adapted to this use. As a consequence, analysis identification, analysis model, fields and properties, analysis shape and analysis control and results are store and use for the model request. To supply the SDM, each transitional created model, all along the process of the figure 4, is stored and managed in configuration. The validation of the model is obtained by comparing the experimental results and the numerical results. Based on this comparison, it is possible to confirm the specifications made at the beginning of the process.

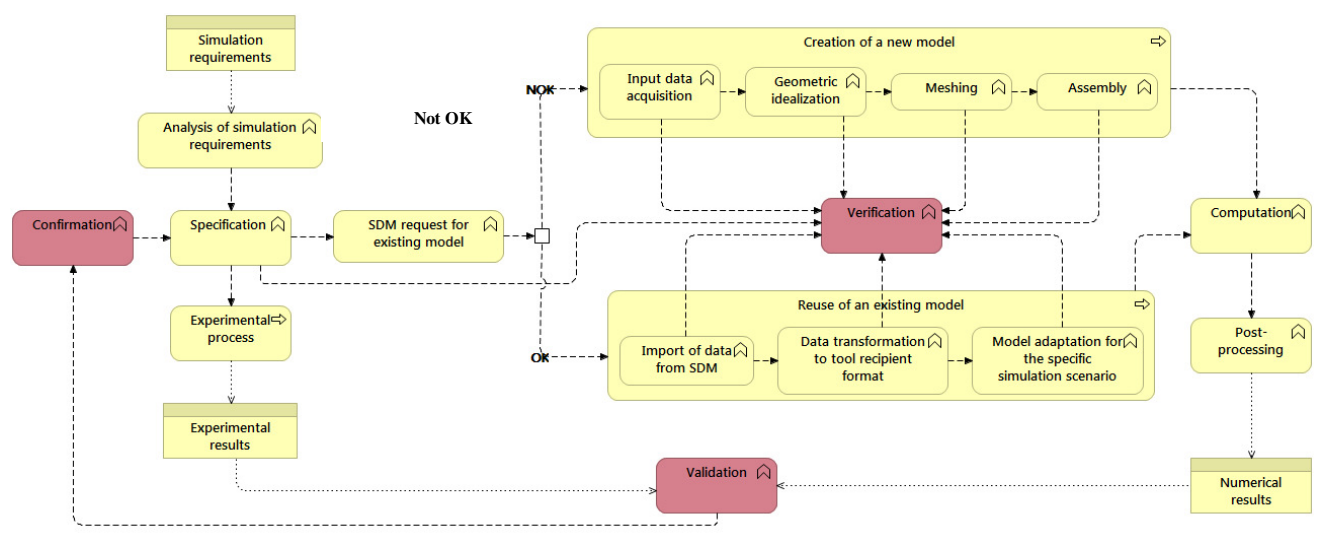

Fig. 4. Simulation process integrating the reuse of models

\section{Conclusion}

The reuse of models in the simulation process is an industrial challenge which will allow the optimization of product development process. The use of SDM systems in accordance to international standard is a necessity to allow this enhancement. This paper proposes an approach enabling the reuse of model based on a combination of SLM approach and V\&V methodology built on international standards. In this framework, a reuse process of models has been proposed.

In future works, this approach will be detailed further. In addition, this process will be developed on industrial practices, and the standard capacity for reuse requirements will be tested.

Acknowledgments. This research work has been carried out under the leadership of the Technological Research Institute SystemX, and therefore granted with public funds within the scope of the French Program "Investissements d'Avenir". 


\section{References}

[1] T. Van Nguyen, F. Ferru, P. Guellec, and B. Yannou, "Engineering data management for extended enterprise - Context of the european VIVACE project", in PLM-SP2, pp. 338 - 348, 2006.

[2] J. Le Duigou, A. Bernard, N. Perry, J.C. Delplace, "Generic PLM system for SMEs: Application to an equipment manufacturer", International Journal of Product Lifecycle Management, vol. 6, no 1, pp. 51-64, 2012.

[3] N. Figay, D. Tchoffa, P. Ghodous, E. Exposito, and A. El Mhamedi, "Dynamic Manufacturing Network, PLM Hub and Business standards testbed", in Proceedings of the I-ESA Conferences, vol. 7, pp. 453-463, 2428 March 2014, Albi, France.

[4] CIMdata, "Simulation Lifecycle Management - More than data management for simulation", Michigan, 2011.

[5] H. a ElMaraghy, "Changing and evolving products and systems - Models and enablers", in Changeable and reconfigurable manufacturing systems, $\mathrm{H}$. A. ElMaraghy, Ed. Springer London, pp. 25-45, 2009.

[6] P. Lalor, "Simulation Lifecycle Management - Opens a new window on the future of product design and manufacturing", 2007.

[7] G. M. Mocko and S. J. Fenves, "A Survey of Design - Analysis Integration Issues", NISTIR 6996, 2003.

[8] N. Figay, P. Ghodous, M. Khalfallah, and M. Barhamgi, "Interoperability framework for dynamic manufacturing networks", Computers in Industry, vol. 63, no. 8, pp. 749-755, 2012.

[9] S. Charles, G. Ducellier, L. Li, and B. Eynard, "Improvement of 3D data exchanges in the product lifecycle management", in Proceedings of the international conference on Product Lifecycle Management, pp. 507-516, 11-13 July 2005, Lyon, France.

[10] T. Nguyen Van, B. Maille, and B. Yannou, "Digital Mock-Up - Capabilities and implementation in the PLM field", in Proceedings of the international conference on Product Lifecycle Management, pp. 165-175, 10-12 July 2006, Bangalore, India.

[11] M. S. Shephard, M. W. Beall, R. M. O. Bara, and B. E. Webster, "Toward simulation-based design", Finite Elements in Analysis and Design, vol. 40, no. 12, pp. 1575-1598, 2004.

[12] F. Delalondre, C. Smith, and M. S. Shephard, "Collaborative software infrastructure for adaptive multiple model simulation", Computer Methods in Applied Mechanics and Engineering, vol. 199, no. 21-22, pp. 1352-1370, 2010.

[13] I. Assouroko, P. Boutinaud, N. Troussier, B. Eynard, and G. Ducellier, "Survey on standards for product data exchange and sharing: application in CAD/CAE interoperability", International Journal of Design and Innovation Research, vol. 5, no. 1, pp. 9-15, 2010.

[14] O. Balci, "Validation, verification, and testing techniques throughout the life cycle of a simulation study", in Simulation conference proceedings, pp. 215 - 220, 11-14 December 1994, Orlando, USA. 
[15] R. Sibois, T. Määttä, M. Siuko, and J. Mattila, "Early design verification of ITER remote handling systems using digital mock-ups within simulation lifecycle environment", in 25th symposium on fusion engineering (SOFE), pp. 1-6, 10-14 June 2013, San Francisco, USA.

[16] P. G. Maropoulos and D. Ceglarek, "Design verification and validation in product lifecycle", CIRP Annals - Manufacturing Technology., vol. 59, no. 2, pp. 740-759, 2010.

[17] B. H. Thacker, S. W. Doebling, F. M. Hemez, M. C. Anderson, J. E. Pepin, and E. A. Rodriguez, "Concepts of model verification and validation", Los Alamos, 2004.

[18] S. Schlesinger, "Terminology for model credibility", Simulation, vol. 32, no. 3, pp. 103-104, 1979.

[19] W. L. Oberkampf, T. G. Trucano, and C. Hirsch, "Verification, validation, and predictive capability in computational engineering and physics", Applied Mechanics Reviews, vol. 57, no. 5, pp. 345-384, 2004.

[20] R. Scigliano, M. Scionti, and P. Lardeur, "Verification, validation and variability for the vibration study of a car windscreen modeled by finite elements", Finite Elements in Analysis and Design, vol. 47, no. 1, pp. 17-29, 2011.

[21] P. J. Roache, Verification and validation in computational science and engineering. Hermosa, 1998.

[22] AIAA, "R-101A - AIAA recommended practice for the CFD General Notation System - Standard interface data structures", 2005.

[23] ISO, "ISO 10303-209 - Application protocol: multidisciplinary analysis and design", 2014.

[24] A. Calvaire, H. P. de Koning, and P. Huau, "STEP-TAS-177-AP Application protocol: thermal analysis for space", 1998

[25] M. J. Pratt, "Introduction to ISO 10303 - the STEP standard for product data exchange", Journal of Computing and Information Science in Engineering, vol. 1, no 1, pp. 102-103, 2001.

[26] K. A. Hunten, "Design and manufacture of composite material product", in Interoperability for digital engineering systems, FrancoAnge, pp. 61-66, 2014.

[27] ISO, "ISO 10303-242 - Application protocol: managed model-based 3D engineering", 2014.

[28] SAE International, "Aerospace Recommended Practice (ARP) 4754A," 2010.

[29] ASME, "V V 10 - Guide for verification and validation in computational solid mechanics", 2006.

[30] IEEE SA, "1012 - IEEE standard for system and software verification and validation", 2012.

[31] ASME, "V V 20 - Standard for verification and validation in computational fluid dynamics and heat transfer", 2009.

[32] ISO, "ISO 10303-233 - Part 233: Application protocol: systems engineering" 2012.

[33] The Open Group, ArchiMate 2.0 specification. Van Haren Publishing, 2012. 